\title{
A prescrição de psicotrópicos e a reavaliação médica
}

\author{
The psychotropic prescription and the medical evaluation
}

Fernanda Santana Rosa', Maria Tereza Mattos Monteiro², Jucélia Jeremias Fortunato³, Dayani Galato

Prezado editor,

Apresentamos os resultados de um estudo com delineamento transversal que teve por objetivo identificar os fatores relacionados à reavaliação da prescrição médica de psicotrópicos. Foram avaliados os usuários de psicotrópicos atendidos em Unidades Básicas de Saúde Pública do município de Tubarão, localizado ao sul do estado de Santa Catarina. Para o cálculo da amostra, considerou-se uma população infinita, um erro de 5\%, um intervalo de confiança de 95\% e uma prevalência estimada para uso de psicotrópico de 9,9\%².

O desfecho considerado nesta pesquisa foi o período considerado adequado para reavaliação médica após prescrição de um psicotrópico, ou seja, até dois meses, de acordo com a Agência Nacional de Vigilância Sanitária (Anvisa)². Na (re)avaliação médica, supõe-se que a necessidade, a efetividade e a segurança dos psicotrópicos sejam aferidas pelo médico antes da elaboração ou renovação da prescrição. Para identificar os fatores associados ao desfecho, foi adotado o teste do qui-quadrado ( $p<0,05)$. Este estudo foi aprovado por comitê de ética em pesquisa, sendo aplicado o termo de consentimento livre e esclarecido a todos os entrevistados.

Foram entrevistados 137 sujeitos com idade entre 20 e 87 anos, em sua maioria (63,5\%) mulheres. A escolaridade média foi de 7,4 \pm 3,1 anos e a média de renda mensal, de 784,2 \pm 520,6 reais. As indicações mais comuns para prescrições de psicotrópicos foram depressão ( $n=67 ; 48,2 \%)$, ansiedade $(n=61 ; 43,9 \%)$ e insônia $(n=51 ; 36,7 \%)$. Os entrevistados relataram utilizar entre um e três psicotrópicos, apresentando o uso de uma média de 1,2 $( \pm 0,5)$ diferentes medicações dessa classe. Os tipos de psicotrópicos mais comumente prescritos foram os antidepressivos (53,3\%) e os ansiolíticos (31,5\%).

Os usuários de psicotrópicos relataram ter realizado a última avaliação entre um e 15 meses da data da entrevista, com média de intervalo de avaliação de 5,6 meses. Apenas 34 pacientes $(24,8 \%)$ descreveram o intervalo de até dois meses entre a última prescrição e a última reavaliação médica do uso dos psicotrópicos. Segundo os entrevistados, as últimas prescrições foram elaboradas por psiquiatras em somente 11,0\% das situações.

Neste estudo, a maior parte dos pacientes não foi reavaliada após a prescrição de psicotrópicos de acordo com a recomendação vigente, o que levanta o questionamento sobre a maneira como as pessoas estão recebendo as prescrições para a aquisição desses medicamentos. Além disso, a única variável que apresentou associação com a reavaliação periódica adequada foi o diagnóstico referido de depressão $(p<0,001)$. Talvez isso tenha ocorrido em virtude de os médicos julgarem necessária uma avaliação mais frequente da medicação nesses pacientes 6 . Sexo não foi um fator associado ao período adequado de reavaliação médica, embora a literatura ${ }^{3-6}$ descreva maior prevalência de uso de medicamentos psicotrópicos

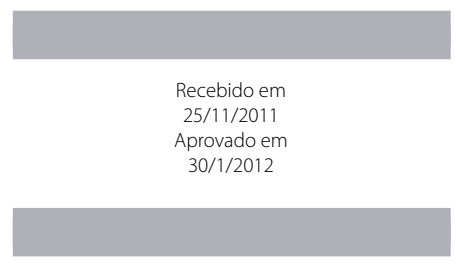

\footnotetext{
1 Universidade do Sul de Santa Catarina (Unisul), Programa de Pós-Graduação em Ciências da Saúde.

2 Unisul, Curso de Farmácia.

3 Unisul, Programa de Pós-Graduação em Ciências da Saúde.

4 Unisul, Núcleo de Pesquisa em Atenção Farmacêutica e Estudos de Utilização de Medicamentos (NAFEUM), Programa de Pós-Graduação em Ciências da Saúde.
} 
em mulheres. Outros fatores como renda, idade e escolaridade do paciente também não se mostraram associados à reavaliação médica periódica.

Constatamos também que, na maioria das vezes, a última prescrição dos psicotrópicos não foi realizada por médicos psiquiatras. Estudos apontam a dificuldade dos médicos, em especial dos generalistas, de diagnosticar e tratar pacientes com transtornos psiquiátricos ${ }^{7}$. Dybwad et al. ${ }^{8}$, em um estudo realizado na Noruega, demonstrou que muitos clínicos-gerais prescrevem medicamentos psiquiátricos apenas por solicitação dos pacientes que já utilizam essas medicações, sem necessariamente realizar uma nova avaliação. Um fenômeno semelhante pode estar ocorrendo em nossa população.

\section{REFERÊNCIAS}

1. Rodrigues MAP, Facchini LA, Lima MS. Modificações nos padrões de consumo de psicofármacos em localidade do sul do Brasil. Rev Saude Publica. 2006;40(1):107-14.
2. Brasil. Ministério da Saúde. Agência Nacional de Vigilância Sanitária. Portaria nº 344, de 12 de maio de 1998. Disponível em: http://www.anvisa.gov.br/legis/portarias/344_98.htm. Acessado em: Ago 8, 2011.

3. Andrade $M$, Andrade R, Santos V. Prescrição de psicotrópicos: avaliaçãa das informações contidas em receitas e notificações. Rev Bras Cienc Farm. 2004;40(4):471-9.

4. Dal Pizzol TS, Branco MMN, Carvalho RMA, Pasqualotti A, Maciel EN, Migott AMB. Uso não médico de medicamentos psicoativos entre escolares do ensino fundamental e médio no sul do Brasil. Cad Saude Publica. 2006;22(1):109-15.

5. Muza GM, Bettio H, Muccillo G, Barbieri MA. Consumo de substâncias psicoativas por adolescentes escolares de Ribeirão Preto, SP (Brasil). I - prevalência do consumo por sexo, idade e tipo de substância. Rev Saude Publica. 1997;31(1):21-9.

6. Nappo SA, Tabach R, Noto AR, Galduroz JCF, Carlini EA. Use of anorectic amphetamine-like drugs by Brazilian women. Eat Behav. 2002;3(2):153-65.

7. Helena E, Lasagno B, Vieira R. Prevalência de transtornos mentais não psicóticos e fatores associados em pessoas com hipertensão arterial sistêmica e/ou diabetes mellitus em Unidades de Saúde da Família em Blumenau, Santa Catarina. Rev Bras Med Fam Com. 2010;5(17):42-7.

8. Dybwad TB, Kjolsrod L, Eskerud J, Laerum E. Why are some doctors high-prescribers of benzodiazepines and minor opiates? A qualitative study of GPs in Norway. Farm Pract. 1997;14(5):361-8. 